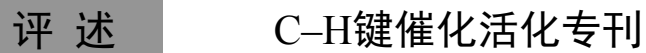

\section{选择性催化混合单体制备多组分聚酯的研究进展}

\author{
周延川，庞烜"，陈学思 ${ }^{*}$ \\ 中国科学院长春应用化学研究所, 中国科学院生态环境高分子材料重点实验室, 长春 130022 \\ *通讯作者, E-mail: xpang@ciac.ac.cn; xschen@ciac.ac.cn \\ 收稿日期: 2020-09-15; 接受日期: 2020-11-11; 网络版发表日期: 2021-01-27 \\ 国家自然科学基金(编号: 21574124)资助项目
}

\begin{abstract}
摘要以聚乳酸和聚碳酸酯等为代表的生物可降解高分子材料已被应用于包装材料和生物医用等领域。然而 受链段结构的影响, 这类均聚物材料在实际应用中受到了一定限制. 将不同性质聚合物链段通过共价键连接形成 的嵌段共聚物具有组分均匀、性能可控等优点, 是优化材料性能的一种方法. 本文概述了环酯、环氧化物和 $\mathrm{CO}_{2}$ (或环状酸酐)的多元共聚反应合成嵌段聚酯的研究进展. 首先介绍了多元共聚反应中链增长机理和判断嵌段共聚 物结构的表征方法, 进一步讨论了催化体系-聚合物结构的关系和聚合物材料的性能表征.
\end{abstract}

关键词选择性催化, 开环聚合反应, 聚酯, 多元共聚物

\section{1 引言}

作为一类潜在替代石油基传统塑料的环保材料, 生物可降解高分子材料的研究和推广已经成为社会 各界备受关注的领域之一 ${ }^{[1,2]}$. 聚酯是一类重要的化学 合成生物可降解高分子材料，常见的材料有聚乳酸 (PLA)、聚已二酸-对苯二甲酸-丁二醇酯(PBAT)、聚 丁二酸丁二醇酯(PBS)和聚己内酯(PCL)等. 其中，聚 乳酸是以乳酸作为重复单元的可再生材料, 乳酸来源 于玉米和甜菜等农作物资源, 具有优异的生物相容 性、热力学性质和拉伸强度, 是替代传统石油基不可 降解塑料的性价比最高的高分子材料 ${ }^{[3 \sim 5]}$. 此外, 聚碳 酸丙烯酯 $(\mathrm{PPC})$ 等是以 $\mathrm{CO}_{2}$ 为 $\mathrm{C} 1$ 原料来源的可降解高 分子材料 ${ }^{[6]}$, 具有单体取代结构丰富、价格低廉和低 能耗等特点 ${ }^{[7,8]}$. 据统计, 与传统的石化产品相比, 合 成聚碳酸酯材料可以减少 $20 \%$ 的石化原料消耗，并减
少大约 $20 \%$ 的二氧化碳排放. 这些可降解材料虽然已 应用于包装材料、餐具和生物医用等领域，但是与传 统的聚乙烯、聚苯乙烯等不可降解材料相比, 其性能 仍然有一定的差距，从而限制了他们的进一步推广 应用。

高分子材料通常采用共混改性或表面改性等方 法改善材料性能，这种方法虽然操作简便，但是所制 备材料的稳定性相对较差. 为了合成组分均一、性能 可控的聚合物，科研工作者设计合成了多元共聚物， 调节聚合物中单体插入的序列实现从分子尺度上对 材料性能进行调控 ${ }^{[9 \sim 13]}$. 这类材料的性能无法从对应 的嵌段组分或其共混物中获得, 因此具有极大的实用 价值.

经过近 50 年的发展, 科研工作者已经从催化体系 和聚合机理等方面对PLA、PCL、PPC等聚(碳酸)酯 的制备作了系统的研究, 其中美国的Coates教授、英

引用格式: Zhou Y, Pang X, Chen X. Recent progress in the preparation of multicomponent polyesters by selective catalysis from mixed monomers. Sci Sin Chim, 2021, 51: 144-153, doi: 10.1360/SSC-2020-0181 
国的Williams教授和大连理工大学的吕小兵教授等 对该领域做了重要贡献. 同时, 由相同开环聚合反应 机理的多组分单体制备嵌段聚酯(如聚乳酸-聚己内 酯共聚物 (PLA-PCL) 和聚乳酸-羟基乙酸共聚物 (PLGA)等)也引起人们的关注 ${ }^{[11,14]}$, 这种方法具有简 捷调节嵌段聚合物的分子结构、组成和分子量等优 点. 然而环内酯单体和交酯单体的取代结构较少, 导 致所得材料性能的调节范围受限. 环氧化物和环状酸 酐的丰富单体取代结构赋予了对应交替共聚物材料 丰富的性能, 将其与聚乳酸或聚己内酯等链接为嵌段 共聚物可以整合对应链段的性能, 扩展材料的应用领 域. 在环酯、环氧化物和 $\mathrm{CO}_{2}$ (或环状酸䣶)的多组分
聚合反应中, 调节反应条件和催化体系可以得到无规 或(多)嵌段聚酯(示意图1). 其中，2014年Williams教 授课题组开创性地报道了在“一锅法”多组分聚合反 应中, $\mathrm{CO}_{2}$ 调节环氧化物、 $\mathrm{CO}_{2}$ 和环内酯的可控共聚 反应, 实现了链段结构可控嵌段聚合物的制备, 具有 操作简便、聚合物结构可以调控等优点 ${ }^{[12]}$. 本文概 括了近年来在催化环酯、环氧化物与 $\mathrm{CO}_{2}$ (或环状酸 䣶)的多元共聚反应合成多嵌段聚酯方面的研究进 展. 首先介绍了在多组分共聚反应中的链增长机理, 进一步介绍了催化体系设计思路和聚合物性能表征 等方面的研究工作, 并对该体系的未来发展进行了 展望.

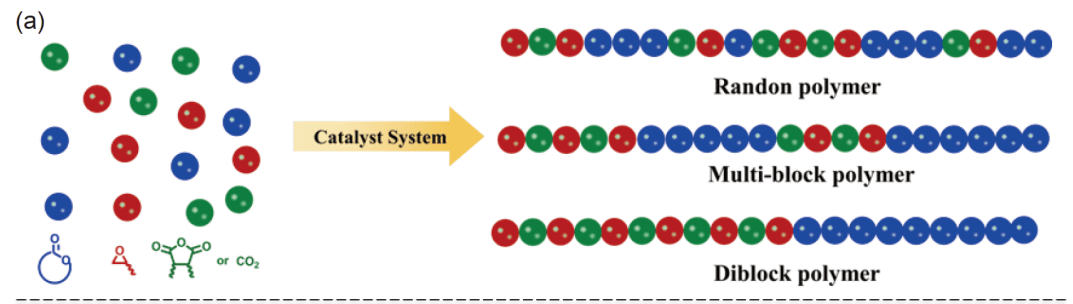

(b)
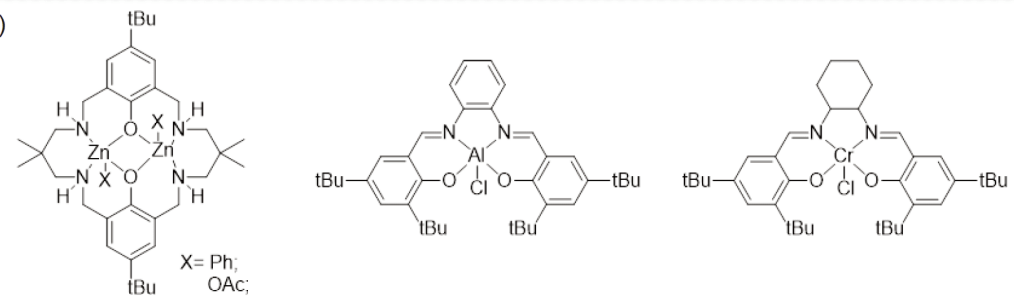

Robson Zn(X)

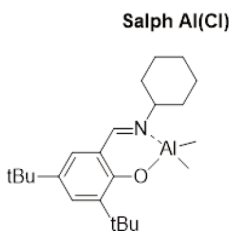

Salen $\mathrm{Cr}(\mathrm{Cl})$

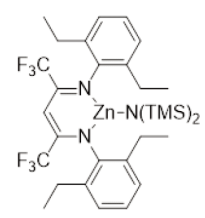

$\mathrm{BDI}^{\mathrm{CF}}-\mathrm{Zn}-\mathrm{N}(\mathrm{TMS})_{2}$

Half-Salen $\mathrm{Al}(\mathrm{Cl})$

$\mathrm{Et}_{3} \mathrm{~B} / \mathrm{DBU}$ pair

${ }^{\mathrm{t} B u P} \mathrm{P}_{1}$

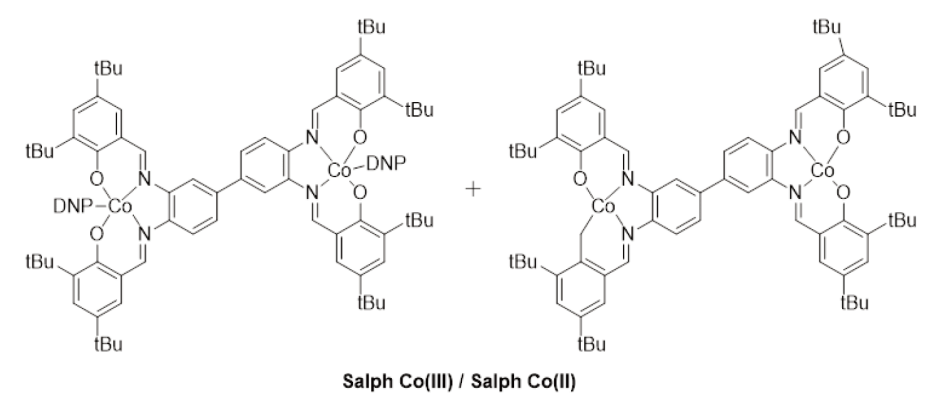

示意图 1 (a) 选择性催化多组分单体制备聚酯示意图; (b) 催化合成(多)嵌段聚合的催化体系(网络版彩图)

Scheme 1 (a) Scheme of preparation of polyesters by selective polymerization of multicomponent monomers; (b) catalyst systems for the preparation of (multi)block polymer (color online). 


\section{2 三元共聚反应的机理及嵌段共聚物判断 依据}

聚乳酸和聚己内酯等聚酯类材料通常是由其对应 的环状单体通过开环聚合反应制备得到 ${ }^{[15 ~ 18]}$, 在此反 应过程中，催化剂决定着聚合反应的活性和聚合物链 段结构. 目前, 席夫碱金属(铝、镁、锌、铁和钛等)配

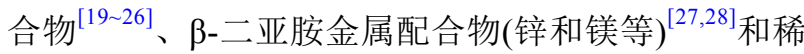
土配合物 ${ }^{[29 ~ 31]}$ 等有机金属配合物对开环聚合反应表 现出对反应进程和聚合物分子量可控、分子量分布 窄, 以及聚合物立体结构可控等优点, 其聚合反应机理 和材料性能已经得到深入的研究 ${ }^{[14,15,32 ~ 34]}$.

另一方面, 采用环氧化物 $/ \mathrm{CO}_{2}$ 催化共聚反应合成 聚碳酸酯是一种废弃资源高值化利用的绿色反应路 线. 取代结构丰富的环氧类单体赋予了聚碳酸酯材料 丰富的链段结构和物理/化学性质 ${ }^{[35 ~ 37]}$. 例如, 将环氧 单体由环氧丙烷 $(\mathrm{PO})$ 替换为环氧环己烷 $(\mathrm{CHO})$ 可以将 材料的玻璃化转变温度 $\left(T_{\mathrm{g}}\right)$ 从 $42^{\circ} \mathrm{C}$ 提高至 $118^{\circ} \mathrm{C}^{[38]}$; 带 有乙烯基等官能团的环氧单体为聚合物的后修饰提供 了活性位点 ${ }^{[39,40]}$. 此外, 该共聚反应对环氧化物和环状 酸酐也具有普适性 ${ }^{[41]}$. 由环氧化物与环状酸酐的共聚 反应可以得到链段结构丰富、性能可调的聚酯材 料 ${ }^{[42,43]}$, 英国的Williams 教授 ${ }^{[41]}$ 和美国的Coates 教授 ${ }^{[44]}$ 已经详细综述了这类聚合反应的单体结构、催化剂类 型、聚酯链段结构及其物理性能.

许多催化体系可以分别适用于环酯开环聚合反应 和环氧化物 $/ \mathrm{CO}_{2}$ (或环状酸䣶)共聚反应, 但是这两类 聚合反应的链增长机理的差异导致不同的催化体系和 单体通过“一锅法”共聚反应可以得到不同链段结构的 聚合物. 以金属配合物催化多元共聚反应的反应机理 为例说明(图1): 环酯单体连续在金属-烷氧键间配位 插入、开环形成聚酯链段; 而对于环氧化物/ $\mathrm{CO}_{2}$ (或 环状酸䣶)的共聚反应, $\mathrm{CO}_{2}$ (或环状酸䣶)单体在金属烷氧键间配位的配位能力强于环氧化物的配位能力, 得到金属-碳酸酯键(或金属-酯键), 之后环氧化物的插 入再次形成金属-烷氧键，通过 $\mathrm{CO}_{2}$ (或环状酸䣶)和环 氧化物的连续交替插入形成聚碳酸酯(或聚酯)链段. 在这三类聚合反应中都有金属-烷氧基键中间体，这为 桥连不同类型的聚合反应制备多元聚合物提供了机 会. 不同单体在金属-烷氧基键间的配位能力差别较大 (通常其配位能力的强度比较为环状酸䣶 $>\mathrm{CO}_{2}>$ 环

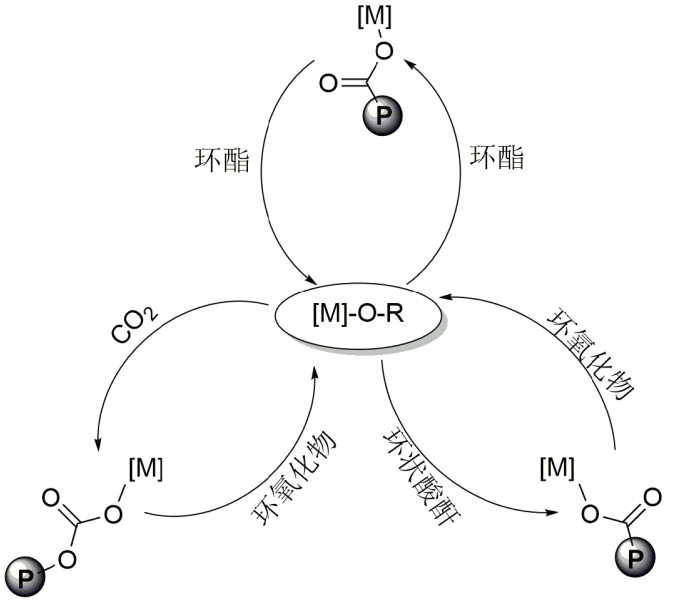

图 1 金属配合物催化多组分共聚反应的链增长机理 Figure 1 Chain-grown mechanism of multicomponent polymerization catalyzed by metal complexes.

酯 ${ }^{[45-49]}$, 导致多元聚合反应易生成嵌段共聚物或梯形 共聚物; 而在一些催化反应体系中单体的配位能力相 近, 使聚合物链段单元在统计意义上呈无规分布.

在多组分单体的聚合反应后需要进一步分析聚合 物的结构, 以判断是否为共聚物. 常用于确定这类聚合 反应得到嵌段共聚物的依据有: (1) 聚合物分子量随聚 合反应进程逐渐增加，同时维持稳定的分子量分布; （2）聚合反应不同阶段端基基团的转换; (3) 聚合物的 扩散系数表征(通常采用DOSY NMR核磁方法测试); (4) 不同聚合物链段间连接基团的表征; (5) 酯交换反 应; (6) 材料的热学和机械性能变化; (7) 聚合物采用溶 剂洗涤保持稳定的组分比例 ${ }^{[50]}$.

\section{3 多嵌段共聚物}

由于环酯开环聚合反应和环氧化物 $/ \mathrm{CO}_{2}$ 共聚反应 的过程中都有烷氧基中间体, 因此可以采用链转移方 法或单体的竞争插入方法将两类聚合物链结合在一 起, 形成多嵌段共聚物.

2015年, 中山大学的孟跃中等 ${ }^{[51]}$ 以己二酸锌为催 化剂, 采用“一步一锅法”催化 $\mathrm{PO} 、 \mathrm{CO}_{2}$ 和左旋丙交酯 (LLA)的三元共聚反应, 由于LLA的配位-开环活性较 高, 得到了一种具有较长LLA链段的三元共聚物. 在 反应过程中, LLA单体的引入可显著提高催化剂的催 化活性，同时对聚碳酸酯单元的选择性也有明显的提 
升. 在PPC聚合物中引入聚乳酸链段, 不仅可以提高材 料的热稳定性，同时其晶体结构也赋予了材料优异的 力学性能.

2015年, 浙江大学的张兴宏等 ${ }^{[52]}$ 设计了锌-钴双金 属氧化络合物( $\mathrm{Zn}-\mathrm{Co}(\mathrm{III}) \mathrm{DMCC}) /$ 辛酸亚锡 $\left(\mathrm{Sn}(\mathrm{Oct})_{2}\right)$ 二元体系用于催化 $\mathrm{CHO} / \mathrm{CO}_{2} / \varepsilon$-己内酯( $\varepsilon-\mathrm{CL}$ )的共聚反 应体系. $\mathrm{Zn}-\mathrm{Co}$ (III) $\mathrm{DMCC}$ 是一种对 $\mathrm{CHO} / \mathrm{CO}_{2}$ 共聚反 应具有高催化活性的催化剂, 而对 $\varepsilon-C L$ 无催化活性; 与之相反, $\mathrm{Sn}(\mathrm{Oct})_{2}$ 只能催化 $\varepsilon-\mathrm{CL}$ 的开环聚合反应. 该 聚合反应体中的两类催化剂分别催化 $\mathrm{CHO} / \mathrm{CO}_{2}$ 的共 聚反应和 $\varepsilon-C L$ 的开环聚合反应的同时，两类不同的烷 氧基中间体在 $\mathrm{Zn}-\mathrm{Co}(\mathrm{III}) \mathrm{DMCC}$ 和 $\mathrm{Sn}(\mathrm{Oct})_{2}$ 间的缓慢 链交换反应将聚碳酸环己烯酯( $\mathrm{PCHC}$ ) 链段和聚己内 酯( $\mathrm{PCL}$ )链段通过共价键连接, 得到多嵌段共聚物.

2018年, 陈学思课题组 ${ }^{[53]}$ 报道了Salen-Co(II)/ Salen-Co(III)/双 (三苯基膦)氯化铵(PPNCl)的三组分体 系催化环氧化物、 $\mathrm{CO}_{2}$ 和 $\mathrm{LA}$ 三元共聚反应合成多嵌 段聚合物的工作。在该三组分单体的共聚反应中, Salen-Co(III)催化环氧化物/ $\mathrm{CO}_{2}$ 的共聚反应, 而对LA 是催化惰性的 ${ }^{[54]}$; 与之相反, Salen-Co(II)对聚碳酸酯 链段的选择性非常差, 但可以催化LA的开环聚合反 应. 不断增长的两类聚合物链末端在Salen-Co(III)和 Salen-Co(II)之间链转移和持续的链增长反应, 将 PLLA和PPC链段连接在一起, 形成多嵌段共聚物(链 转移和链增长机理见图2), 该反应机理对不同配体结 构的钴配合物和不同取代结构的环氧单体都具有普适 性(图2).

\section{4 序列可控嵌段聚合物}

核酸、蛋白质等生物大分子中单体序列的精确控 制是导致生物体多样性、复杂性和适应性的关键因素 之一. 聚合物链上的单元序列决定了材料的微观和宏 观性质，如将自然界大分子的结构精确转化为合成高 分子化学中单体序列的可控性, 对于聚合物结构的多 样性和精细定制聚合物性能十分重要. 早期通常是采 用多步法合成结构精确的嵌段共聚物 ${ }^{[55]}$, 例如，吕小 兵教授和Darensbourg 教授等 ${ }^{[56]}$ 采用Salen-Co(III)/ $\mathrm{PPNCl}$ 催化氧化苯乙烯 $/ \mathrm{CO}_{2}$ 聚合反应合成羟基末端的 聚碳酸酯, 再进一步在 1,8 -二氮杂二环十一碳-7-烯 (DBU)的催化作用下引发LA的开环聚合反应，得到嵌

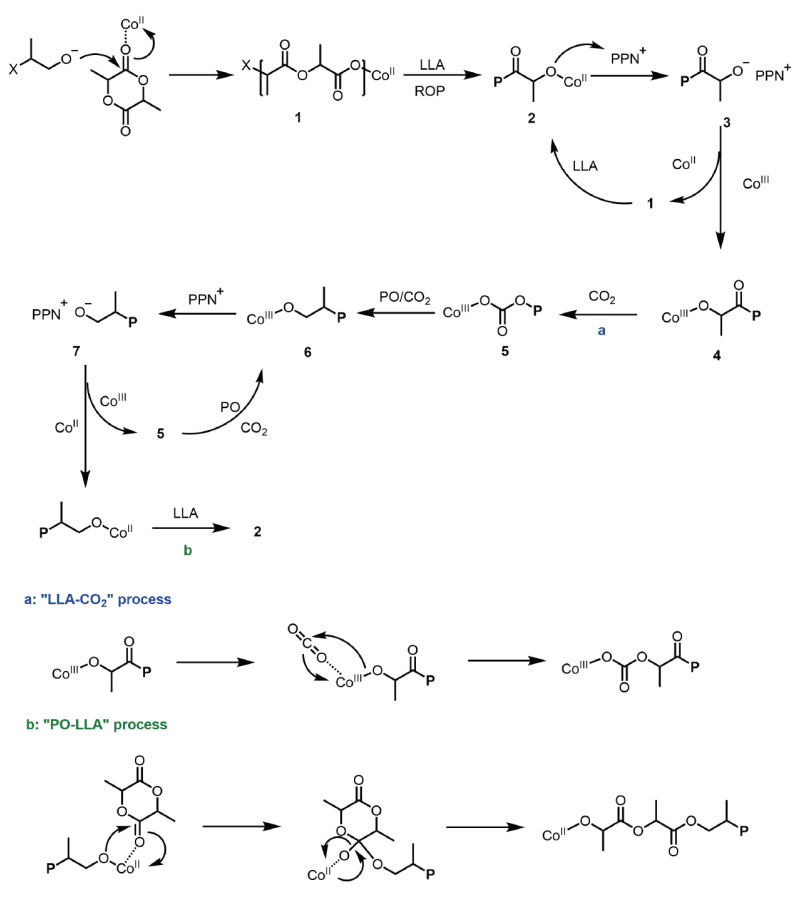

图 2 Salen-Co(II)/Salen-Co(III)/PPNCl三组分体系催化 $\mathrm{PO} 、 \mathrm{CO}_{2}$ 和 $\mathrm{LA}$ 三元共聚反应的链增长机理(网络版彩图)

Figure 2 The proposed propagation mechanism for the terpolymerization of $\mathrm{PO}, \mathrm{CO}_{2}$ and LA catalyzed by Salen-Co(II)/Salen-Co(III)/ $\mathrm{PPNCl}$ (color online).

段共聚物。这种方法可以得到链段精确的聚合物，但 操作步骤烦琐, 增加了时间和人工成本.

近年来，科研工作者开发了一些利用氧化还原 ${ }^{[5]}$ 和光 ${ }^{[58]}$ 等外部调制的方案在线切换聚合进程的策略. 而一种更简单的方法是自切换聚合策略，即在没有任 何外部刺激的情况下实现一步选择性转换聚合进程. 在混合单体原料中, 一种单体是否存在会影响到另一 种单体的反应，这种方法不需要单体或活化剂的连续 添加和反应条件的改变即可实现结构精确嵌段共聚物 的制备. 以下介绍近几年在环酯、环氧化物和 $\mathrm{CO}_{2}$ (或 环状酸䣶)的混合单体中实现序列可控的嵌段聚合物 方面的研究进展.

\section{1 有机金属配合物催化体系}

2014年, 英国的Williams教授课题组 ${ }^{[59]}$ 选用Robson型配体与醋酸锌反应合成的双核锌配合物，催化 $\mathrm{CHO} 、 \mathrm{CO}_{2}$ 和 $\varepsilon-\mathrm{CL}$ 的三组分聚合反应. 该配合物可以 分别催化 $\mathrm{CHO} / \mathrm{CO}_{2}$ 的共聚反应和 $\varepsilon-\mathrm{CL}$ 的开环聚合反 应. 然而当反应体系中存在 $\mathrm{CO}_{2}$ 时, $\varepsilon-\mathrm{CL}$ 无法开环插入 


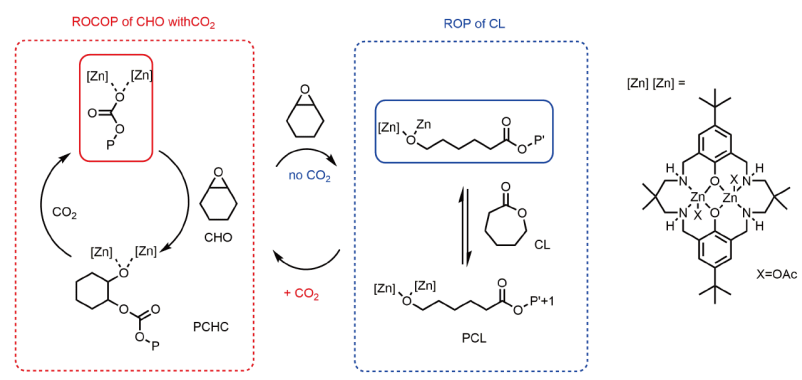

图 3 双核 $\mathrm{Zn}$ 配合物选择性催化 $\mathrm{CHO} / \mathrm{CO}_{2} / \varepsilon-\mathrm{CL}$ 三元共聚反 应示意图(网络版彩图)

Figure 3 Scheme of terpolymerization of $\mathrm{CHO} / \mathrm{CO}_{2} / \varepsilon-\mathrm{CL}$ catalyzed by dinuclear $\mathrm{Zn}$ complex (color online).

到链增长进程中，释放 $\mathrm{CO}_{2}$ 才能激活开环聚合反应(图 3). 他们通过简单的调节反应体系中的气氛实现了嵌 段共聚物的制备. 随后，该课题组报道了该双核锌配 合物催化CHO、邻苯二甲酸䣶 $(\mathrm{PA})$ 和 $\varepsilon$ - 癸内酯 $(\varepsilon-\mathrm{DL})$ 的三元共聚反应 ${ }^{[60]}$. 聚合体系首先进行的是 $\mathrm{CHO} / \mathrm{PA}$ 共聚反应，待 $\mathrm{PA}$ 完全耗尽后则立即转换为 $\varepsilon-D L$ 的开环 聚合反应. 该聚合体系表现出活性聚合的特点, 连续投 料可以得到链段长度可控、窄分子量分布的多嵌段聚 酯化合物.

在以上研究的基础上，该课题组 ${ }^{[45]}$ 采用实验和密 度泛函理论相结合的方法比较了双核锌配合物催化多 组分共聚反应中的单体选择性插入的机理. 如图4所
示, $\mathrm{CO}_{2}$ (或PA)在锌-烷氧基中间体插入的能垒差远低 于 $\varepsilon-C L$ 的能垒差, 因此 $\mathrm{CO}_{2}$ (或PA)会优先插入到链末 端，这与反应体系中存在 $\mathrm{CO}_{2}$ 或PA时无 $\varepsilon-\mathrm{CL}$ 开环插入 的实验现象相符; 而在 $\mathrm{CHO} / \mathrm{CO}_{2} / \mathrm{PA}$ 三元聚合体系中, $\mathrm{CO}_{2}$ 与 $\mathrm{PA}$ 在插入锌-烷氧基中间体的能垒差虽然接近, 但是由于PA的自由能较低, 只有在PA完全消耗尽后才 会进行 $\mathrm{CHO} / \mathrm{CO}_{2}$ 的共聚反应. 综合以上研究可以得出 结论: 在锌配合物的催化体系中, 单体在锌-烷氧基中 间体间插入顺序为 $\mathrm{PA}>\mathrm{CO}_{2}>\varepsilon-\mathrm{CL}$. 这为后续工作研究 提供了理论基础.

2018年，Williams课题组 ${ }^{[61]}$ 报道了采用席夫碱铝 $($ Salen- $\mathrm{Al}(\mathrm{Cl})) / \mathrm{PPNCl}$ 催化PO、PA和外消旋丙交酯 ( $r a c-L A)$ 的三元共聚反应体系, 同样在PA完全消耗后 才转换至 $r a c$-LA的开环聚合反应，得到嵌段共聚物. 该催化反应表现出活性聚合的特点: 在LA基本反应之 后继续补加 $\mathrm{PA}$ 和 $r a c-\mathrm{LA}$, 所得聚合物的分子量继续增 加且分子量分布保持稳定，最终能得到窄分子量分布 $(D=1.21)$ 的二十七嵌段共聚物.

$\beta$-二亚胺锌(BDI-Zn)配合物在催化环酯的开环聚 合反应和环氧化物 $/ \mathrm{CO}_{2}$ 共聚反应中表现出优异的催化 活性和聚合物结构选择性 ${ }^{[46,62 ~ 64]}$. 慕尼黑工业大学的 Rieger课题组 ${ }^{[65]}$ 发现，以 $\beta$-二亚胺锌为催化剂，在“一 锅法”催化 $\beta$-丁内酯(BBL)、 $\mathrm{CHO}$ 和 $\mathrm{CO}_{2}$ 的三元共聚反 应中, 调节反应体系中 $\mathrm{CO}_{2}$ 的压力可实现对聚合物链
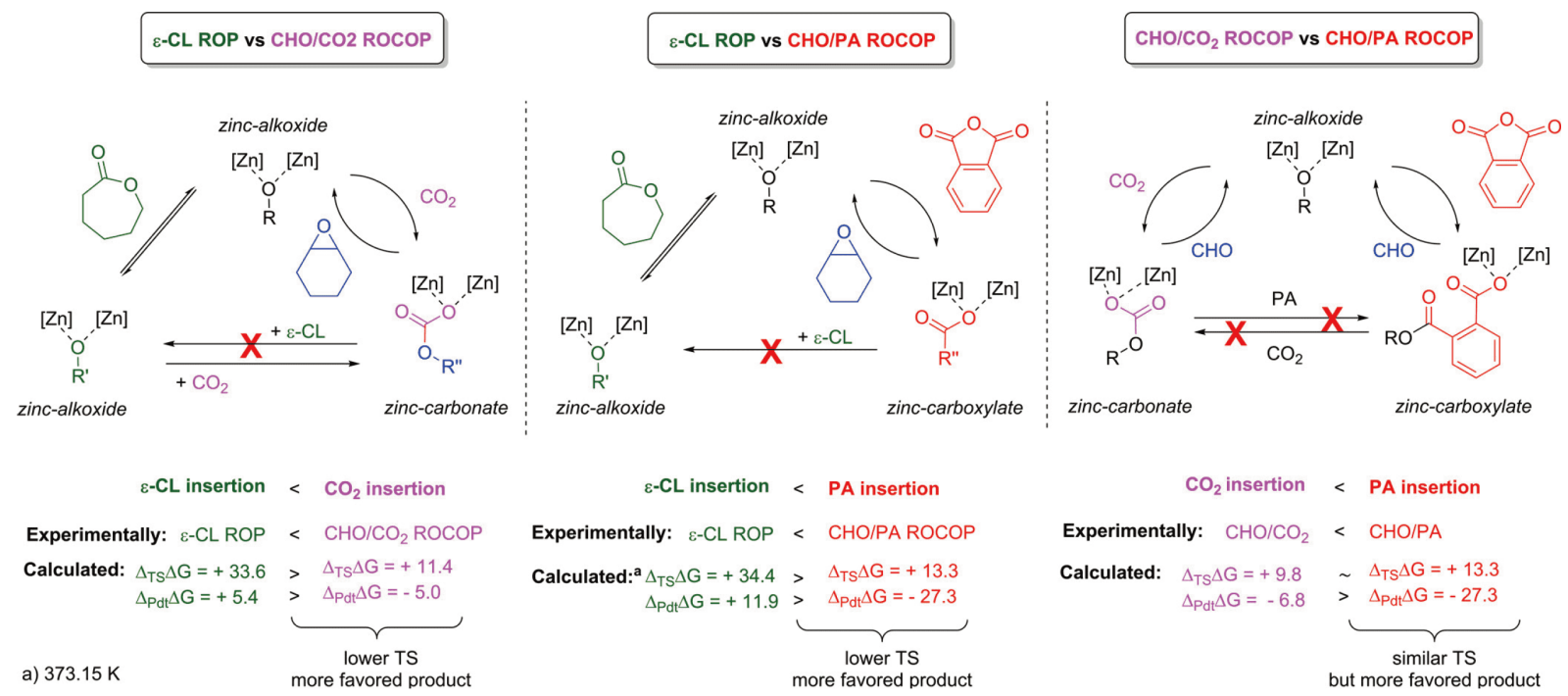

图 4 通过实验和理论方法比较的三种聚合过程中单体的不同排列方式 ${ }^{[45]}$ (网络版彩图)

Figure 4 Three polymerization processes, using various permutations of the monomers, compared by experimental and theoretical methods [45] (color online). 
段结构的调控, 即在体系中充入 $0.3 \mathrm{MPa} \mathrm{CO}_{2}$ 时, $\mathrm{CO}_{2}$ 和 $\mathrm{LA}$ 插入到锌-烷氧基中间体的能垒差别相近, 通过两 类聚合反应在链末端的竞争插入、链增长反应, 得到 具有统计意义上无规序列的三元共聚物; 而 $4 \mathrm{MPa}$ $\mathrm{CO}_{2}$ 只能得到 $\mathrm{CHO} / \mathrm{CO}_{2}$ 的共聚链段, 释放 $\mathrm{CO}_{2}$ 后则转 化为BBL的开环聚合反应, 得到结构明确的两嵌段共 聚物(图5).

2018年, 陈学思课题组 ${ }^{[66]}$ 采用席夫碱铬(Salen-Cr (Cl))/DBU双组分催化剂PO、 $\mathrm{CO}_{2}$ 和LA的共聚反应. 在惰性气体(氩气)氛围中, DBU可以催化丙交酯的开 环聚合反应形成聚乳酸链节，期间无环氧化物的插入; 当反应气氛置换为 $\mathrm{CO}_{2}$ 后, $\mathrm{CO}_{2}$ 在聚合物的烷氧基链末 端快速插入能够完全抑制丙交酯的开环-插入反应, 在 此情况下Salen $\mathrm{Cr}(\mathrm{Cl}) / \mathrm{DBU}$ 催化 $\mathrm{PO}$ 和 $\mathrm{CO}_{2}$ 的开环共聚 反应. 通过调控反应气氛中 $\mathrm{Ar}$ 与 $\mathrm{CO}_{2}$ 间的切换, 实现了 链段长度可控的嵌段聚合物的制备(图6).

此后, 陈学思课题组 ${ }^{[67]}$ 选用半席夫碱铝(half Salen-Al(Me))/PPNCl双组分体系催化环氧化物、环状 酸酐和丙交酯三元共聚反应, 详细分析了聚合进程、

聚合物链段结构及其影响因素, 同时也研究了单体取 代结构对聚合物热性能的影响. 在以环氧氯丙烷 $(\mathrm{ECH}) 、 \mathrm{PA}$ 和LLA为反应模型的研究中发现: 反应的 第一阶段主要是ECH/PA共聚反应，待PA完全消耗后 则进行LA的开环聚合反应，得到两嵌段聚酯; 反应条 件也会影响到链段的规整度，如升高反应温度或降低

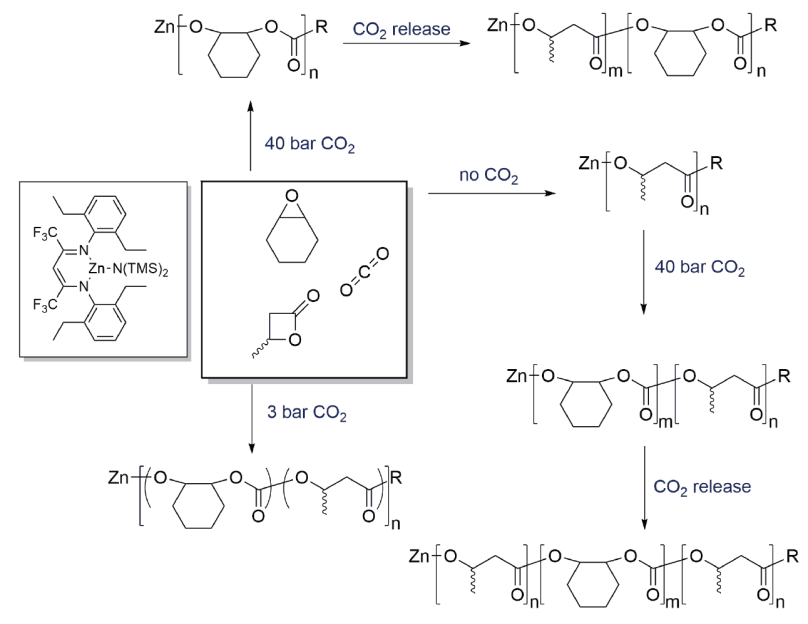

图 $5 \mathrm{CO}_{2}$ 调控的 $\mathrm{CHO} / \mathrm{CO}_{2} / \mathrm{BBL}$ 三元共聚反应 ${ }^{[65]}$ (网络版彩 图)

Figure 5 Chemoselective terpolymerization of $\mathrm{CHO} / \mathrm{BBL} / \mathrm{CO}_{2}$ by $\mathrm{CO}_{2}$ switching [65] (color online).

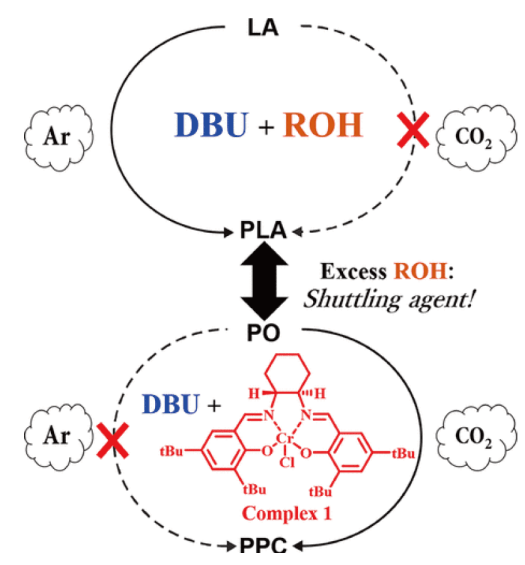

图 6 席夫碱铬/DBU催化制备结构可控嵌段共聚物示意 图 $^{[66]}$ (网络版彩图)

Figure 6 Scheme of preparation of block copolymers with controllable structure catalyzed by salen- $\mathrm{Cr}(\mathrm{Cl}) / \mathrm{DBU}$ binary catalyst [66] (color online).

PPNCl的比例都会导致少量LA插入到 $\mathrm{ECH} / \mathrm{PA}$ 共聚反 应过程中. 此外, 细胞毒性试验发现, 该系列嵌段聚酯 和对应官能化材料几乎没有毒性，这类生物相容性材 料具有生物医学应用的前景.

\section{2 非金属催化体系}

以铝、钴和铬等为活性中心的有机金属配合物在 可降解聚合物的催化合成中虽然表现出优异的催化活 性和链段结构控制性, 然而聚合物的纯化处理仍然有 金属残留，限制了其在组织工程等领域的应用. 为此, 近年来有机碱、硫脲和嗍系等非金属催化剂逐渐引起 了科研工作者的关注. 这类催化体系具有合成简单、 催化活性较高和毒性低等优点.

磷腈类催化剂是一类高活性有机催化剂，对环酯 的开环聚合反应 ${ }^{[68,69]}$ 和环氧化物/环状酸酐的共聚反 应 ${ }^{[70]}$ 都表现出优异的催化性能. ${ }^{t} \mathrm{BuP}_{1}$ 是一种非亲核催 化剂, 在醇作为引发剂的条件下催化环氧化物、环状 酸酐和丙交酯的三元共聚反应, 首先进行的是环氧化 物/酸䣶的共聚反应, 待环状酸酕完全消耗后丙交酯才 在链末端开环插入; 此外, 在催化丙交酯开环聚合反应 过程中加入环状酸䣶单体, 聚合反应会立即转化为环 氧化物/环状酸䣶的共聚反应, 形成链段规整、窄分子 量分布的嵌段共聚物(图7) ${ }^{[71,72]}$.

三乙基硼/有机碱组成的路易斯对是近年来受关 注的一类非金属催化剂, 在环氧化物 $/ \mathrm{CO}_{2}$ 和环氧化物/ 酸䣶的共聚反应中表现出优异的催化性能和选择 


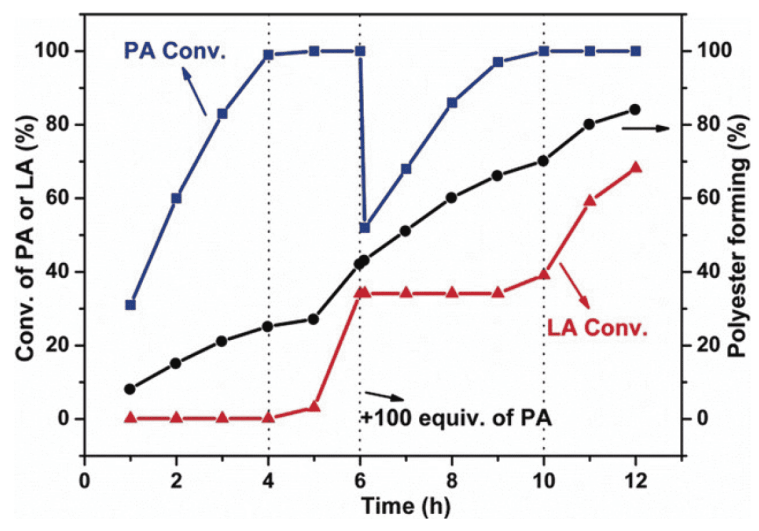

图 $7{ }^{t} \mathrm{BuP}_{1}$ /醇体系催化环氧化物、酸䣶和丙交酯三元共聚 反应的单体转化率随时间变化的示意图 ${ }^{[71]}$ (网络版彩图)

Figure 7 Plots of monomer conversion versus time for the terpolymerization of epoxide, cyclic anhydride and lactide catalyzed by ${ }^{t} \mathrm{BuP}_{1} /$ alcohol [71] (color online).

性 ${ }^{[73,74]}$. 王勇等 ${ }^{[75]}$ 利用三乙基硼 $\left(\mathrm{Et}_{3} \mathrm{~B}\right)$ 和DBU催化 PO、PA和LA的共聚反应，路易斯酸碱对的摩尔比例 决定着聚合物链段的序列结构. 在二元醇做引发剂的 情况下，等摩尔比的 $\mathrm{Et}_{3} \mathrm{~B} / \mathrm{DBU}$ 首先催化 $\mathrm{PO} / \mathrm{PA}$ 的共聚 反应，之后为LA的开环聚合反应，得到三嵌段聚酯; 提高 $\mathrm{Et}_{3} \mathrm{~B} / \mathrm{DBU}$ 摩尔比 $(2: 1)$ 则会在LA消耗尽后继续进 行PO的开环均聚反应，形成聚醚链段，得到五嵌段共 聚物; 进一步增加 $\mathrm{Et}_{3} \mathrm{~B}$ 的摩尔比则会导致LA开环聚合 反应过程中PO的插入, 得到聚乳酸链段中含有聚醚链 节的五嵌段共聚物(图8).

\section{5 嵌段共聚物性能}

与均聚物相比，调节多元共聚物的链段结构可以 对材料的宏观性能进行更高程度的控制, 包括机械性 能、热学性能、相位变化及降解性能等 ${ }^{[9,76,77]}$.

两亲性嵌段聚合物具有自组装特性，在生物材 料、胶黏剂和添加剂等领域有着广泛的应用。这类材 料通常采用端基官能化大分子引发第二段聚合反应等 方法, 实验步骤繁琐并且所得嵌段聚合物的分子量分 布相对较宽. Williams课题组 ${ }^{[78]}$ 报道了含醚段取代基 团的环氧化物、丁二酸䣲和 $\varepsilon-C L$ 的三元共聚反应，一 锅法合成了链段分子量可控、窄分子量分布的嵌段聚 酯. 其中环氧化物/丁二酸䣶链段受侧链聚醚链节的影 响表现出亲水性，与疏水的PCL链段组成双亲性聚酯. 这种材料通过自组装的方式能够进入细胞，同时其降

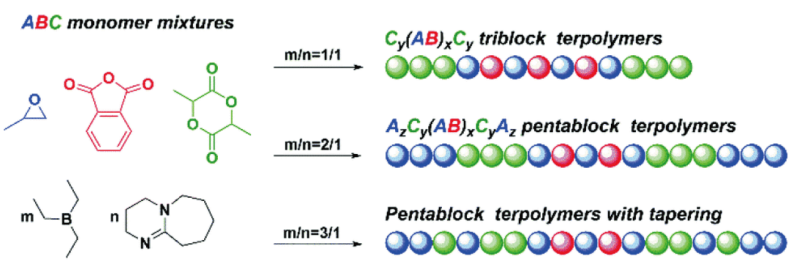

图 $8 \mathrm{Et}_{3} \mathrm{~B} / \mathrm{DBU}$ 对催化PO、PA和LA共混单体的示意图 ${ }^{[75]}$ (网络版彩图)

Figure 8 Scheme of polymerization of PO, PA and LA catalyzed by $\mathrm{Et}_{3} \mathrm{~B} / \mathrm{DBU}$ pair [75] (color online).

解产物具有生物活性, 可以引起细胞分化反应, 诱导 基质细胞增殖和巨噬细胞调亡.

在热学性能和机械性能方面, 嵌段共聚物通过整 合均聚物链段的性质, 可以扩展材料的应用范围. 例 如, 柔性的聚 $\varepsilon$ - 癸内酯(PDL)和刚性的Poly(CHO-alt$\mathrm{PA}$ )链接得到的多嵌段聚酯, 调节链段间的比例实现 了材料从高弹性热塑性弹性体、硬质塑料到形状记忆 聚合物间改变 ${ }^{[79]}$. 这类嵌段聚酯有着比聚乳酸更宽的 工作温度范围，以及与聚苯乙烯类材料相当的机械性 能 ${ }^{[80]}$.

Rieger课题组在研究了 $\mathrm{BBL} 、 \mathrm{CHO}$ 和 $\mathrm{CO}_{2}$ 三元共 聚反应的基础上, 对所得聚合物的性能进行了分析 ${ }^{[81]}$. 由于聚 $\beta$-丁内酯(PHB)链段与聚碳酸酯链段间的相分 离导致嵌段聚合物表现出两个明显不同的 $T_{\mathrm{g}}$ 值, 而无 规共聚物只表现出一个 $T_{\mathrm{g}}$ 值. 在脆性的聚(碳酸环己 烯)聚合物中链接柔性的PHB链后, 嵌段和无规共聚物 的杨氏模量都下降了。聚合物分子量提高到 $100 \mathrm{~kg} / \mathrm{mol}$ 后增加了材料的断裂伸长率, 增强了材料 的力学性能.

\section{6 总结与展望}

在多组分单体的共聚反应中, 单体序列的调控对 所得聚合物的热学性能、降解性和弹性等理化性质有 着重大的影响. 本文介绍了近年来环酯、环氧化物和 $\mathrm{CO}_{2}$ (或环状酸䣶)的多组分共聚反应的重要研究进展, 包括催化体系的组分结构和单体取代等因素对聚合物 结构的影响、聚合反应机理, 以及多元聚酯在自组装 材料和弹性体材料等方面的性能表征. 相比于均聚物, 该聚合反应策略所合成的多元聚酯赋予了材料更宽的 性能可调性.

然而该系列多元聚酯的进一步研究和应用推广的 
道路上仍然有许多问题函待解决，如单体序列的精确 调控、链段结构的立体构型控制、高分子量嵌段的可 控合成和材料结构-性能之间的关系等. 为此, 今后的 研究工作重点应从催化体系的设计着手，使多组分聚
合反应在保持链段序列可控的基础上，实现立体构型 和区域选择性等微观结构的可控调节. 另外, 也需要 进一步总结多元聚酯的单体取代基团、链段结构与材 料性能间的关系, 为材料的应用提供理论指导.

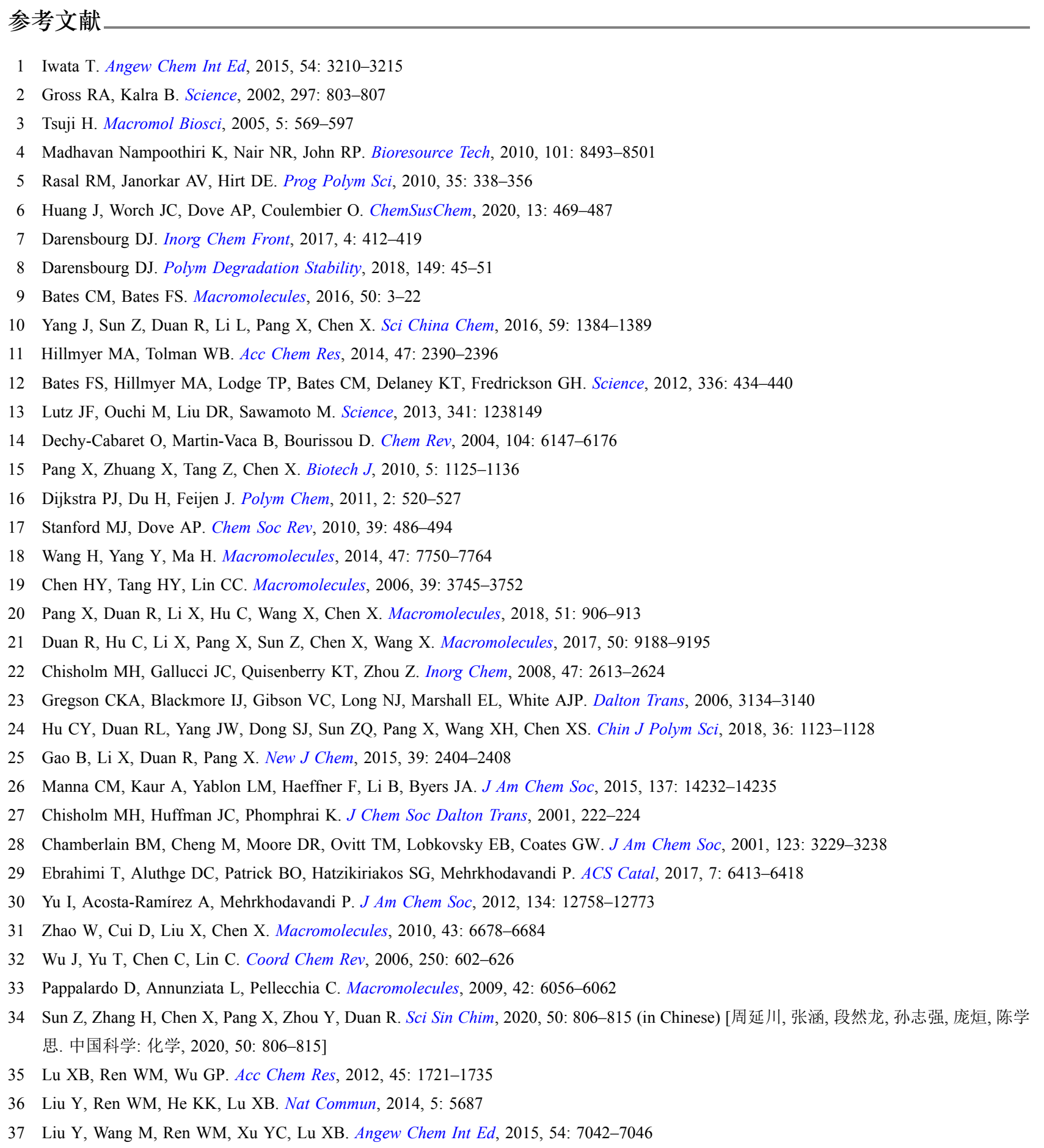


Ren WM, Zhang X, Liu Y, Li JF, Wang H, Lu XB. Macromolecules, 2010, 43: 1396-1402

Darensbourg DJ, Chung WC, Yeung AD, Luna M. Macromolecules, 2015, 48: 1679-1687

Martín C, Kleij AW. Macromolecules, 2016, 49: 6285-6295

Paul S, Zhu Y, Romain C, Brooks R, Saini PK, Williams CK. Chem Commun, 2015, 51: 6459-6479

Yu X, Jia J, Xu S, Lao KU, Sanford MJ, Ramakrishnan RK, Nazarenko SI, Hoye TR, Coates GW, DiStasio Jr. RA. Nat Commun, 2018, 9: 2880

Sanford MJ, Van Zee NJ, Coates GW. Chem Sci, 2018, 9: 134-142

Longo JM, Sanford MJ, Coates GW. Chem Rev, 2016, 116: 15167-15197

Romain C, Zhu Y, Dingwall P, Paul S, Rzepa HS, Buchard A, Williams CK. J Am Chem Soc, 2016, 138: 4120-4131

Jeske RC, Rowley JM, Coates GW. Angew Chem Int Ed, 2008, 47: 6041-6044

Huijser S, HosseiniNejad E, Sablong R, de Jong C, Koning CE, Duchateau R. Macromolecules, 2011, 44: 1132-1139

Darensbourg DJ, Poland RR, Escobedo C. Macromolecules, 2012, 45: 2242-2248

Duan Z, Wang X, Gao Q, Zhang L, Liu B, Kim I. J Polym Sci Part A-Polym Chem, 2014, 52: 789-795

Stößer T, Chen TTD, Zhu Y, Williams CK. Phil Trans R Soc A, 2018, 376: 20170066

Tang L, Luo W, Xiao M, Wang S, Meng Y. J Polym Sci Part A-Polym Chem, 2015, 53: 1734-1741

Li Y, Hong J, Wei R, Zhang Y, Tong Z, Zhang X, Du B, Xu J, Fan Z. Chem Sci, 2015, 6: 1530-1536

Li X, Hu C, Pang X, Duan R, Chen X. Catal Sci Technol, 2018, 8: 6452-6457

Darensbourg DJ, Wu GP. Angew Chem Int Ed, 2013, 52: 10602-10606

Petersen SR, Wilson JA, Becker ML. Macromolecules, 2018, 51: 6202-6208

Wu GP, Darensbourg DJ, Lu XB. J Am Chem Soc, 2012, 134: 17739-17745

Qi M, Dong Q, Wang D, Byers JA. J Am Chem Soc, 2018, 140: 5686-5690

Eisenreich F, Kathan M, Dallmann A, Ihrig SP, Schwaar T, Schmidt BM, Hecht S. Nat Catal, 2018, 1: 516-522

Romain DC, Williams CK. Angew Chem Int Ed, 2014, 53: 1607-1610

Zhu Y, Romain C, Williams CK. J Am Chem Soc, 2015, 137: 12179-12182

Stößer T, Mulryan D, Williams CK. Angew Chem Int Ed, 2018, 57: 16893-16897

Moore DR, Cheng M, Lobkovsky EB, Coates GW. J Am Chem Soc, 2003, 125: 11911-11924

Trofymchuk OS, Daniliuc CG, Kehr G, Erker G, Rojas RS. RSC Adv, 2015, 5: 21054-21065

Chen HY, Huang BH, Lin CC. Macromolecules, 2005, 38: 5400-5405

Kernbichl S, Reiter M, Adams F, Vagin S, Rieger B. J Am Chem Soc, 2017, 139: 6787-6790

Hu C, Duan R, Yang S, Pang X, Chen X. Macromolecules, 2018, 51: 4699-4704

Zhou Y, Hu C, Zhang T, Xu X, Duan R, Luo Y, Sun Z, Pang X, Chen X. Macromolecules, 2019, 52: 3462-3470

Zhao N, Ren C, Li H, Li Y, Liu S, Li Z. Angew Chem Int Ed, 2017, 56: 12987-12990

Hong M, Chen EYX. Angew Chem Int Ed, 2016, 55: 4188-4193

Li H, Zhao J, Zhang G. ACS Macro Lett, 2017, 6: 1094-1098

Ji HY, Wang B, Pan L, Li YS. Angew Chem Int Ed, 2018, 57: 16888-16892

Li H, Luo H, Zhao J, Zhang G. ACS Macro Lett, 2018, 7: 1420-1425

Kummari A, Pappuru S, Chakraborty D. Polym Chem, 2018, 9: 4052-4062

Zhang D, Boopathi SK, Hadjichristidis N, Gnanou Y, Feng X. J Am Chem Soc, 2016, 138: 11117-11120

Zhu S, Wang Y, Ding W, Zhou X, Liao Y, Xie X. Polym Chem, 2020, 11: 1691-1695

Weiss J, Li A, Wischerhoff E, Laschewsky A. Polym Chem, 2012, 3: 352-361

Hao Z, Li G, Yang K, Cai Y. Macromol Rapid Commun, 2013, 34: 411-416

Zhu Y, Poma A, Rizzello L, Gouveia VM, Ruiz-Perez L, Battaglia G, Williams CK. Angew Chem Int Ed, 2019, 58: 4581-4586

Zhu Y, Radlauer MR, Schneiderman DK, Shaffer MSP, Hillmyer MA, Williams CK. Macromolecules, 2018, 51: 2466-2475

Gregory GL, Sulley GS, Carrodeguas LP, Chen TTD, Santmarti A, Terrill NJ, Lee KY, Williams CK. Chem Sci, 2020, 11: 6567-6581

Kernbichl S, Reiter M, Mock J, Rieger B. Macromolecules, 2019, 52: 8476-8483 


\title{
Recent progress in the preparation of multicomponent polyesters by selective catalysis from mixed monomers
}

\author{
Yanchuan Zhou, Xuan Pang ${ }^{*}$, Xuesi Chen ${ }^{*}$ \\ Key Laboratory of Polymer Ecomaterials, Changchun Institute of Applied Chemistry, Chinese Academy of Sciences, Changchun 130022, China \\ *Corresponding authors (email: xpang@ciac.ac.cn; xschen@ciac.ac.cn)
}

\begin{abstract}
Biodegradable polymer materials, such as polylactide and polycarbonate, have been used in packaging materials and biomedicine, etc. However, these homopolymeric materials are limited in the practical applications due to the influence of chain structures. Synthesizing block polymers with designed polymer segments is a method to optimize material properties, which has many advantages including uniform components and controllable properties. This review outlined the research progress in the perparation of block polyesers from cyclic esters, epoxides and $\mathrm{CO}_{2}$ (or cyclic anhydrides). We introduced the chain growth mechanism and the methods of judging block copolymer structures. The relationship between catalytic systems-polymer structures, and materials' properties were further discussed.
\end{abstract}

Keywords: selective catalysis, ring-opening polymerization, polyester, multi-block polymer

doi: $10.1360 /$ SSC-2020-0181 